\author{
Nota científica \\ (Short communication)
}

\title{
ABUNDANCIA Y DENSIDAD DE JAGUAR (PANTHERA ONCA) EN EL APFF MESETA DE CACAXTLA, SINALOA, MÉXICO
}

\section{JAGUAR (PANTHERA ONCA) ABUNDANCE AND DENSITY IN THE APFF MESETA DE CACAXTLA, SINALOA, MEXICO}

\author{
Helí CORONEL-ARELlano, Nalleli E. LARA-DÍAZ y Carlos A. LÓPEZ-GONZÁlez \\ Laboratorio de Zoología, Facultad de Ciencias Naturales, Universidad Autónoma de Querétaro. Av. De las \\ Ciencias S/N, Col. Juriquilla, Querétaro, México. C.P. 76230. E-mails: $<$ heli.coronelarellano@gmail.com>, $<$ lara. \\ nalleli@gmail.com>,<cats4mex@gmail.com> \\ Recibido: 18/02/2016; aceptado: 30/09/2016 \\ Editor responsable: Vinicio Sosa
}

Coronel-Arellano, H., Lara-Díaz, N. E. y López-González, C. A. (2017) Abundancia y densidad de jaguar (Panthera onca) en el APFF Meseta de Cacaxtla, Sinaloa, México. Acta Zoológica Mexicana (n.s.), 33(1), 116-119.

RESUMEN. Existe poca información acerca de las poblaciones del jaguar en Sinaloa, México, ya que sólo se cuenta con registros anecdóticos y entrevistas acerca de la presencia de este félido. El objetivo de este estudio fue generar información acerca de la abundancia y densidad del jaguar en el área natural protegida Meseta de Cacaxtla. Para detectar al jaguar se usaron trampas cámara. La densidad obtenida fue de 1.59 ind./100 km² y 15 jaguares para el área natural protegida. Consideramos que el área natural protegida es una región importante para las poblaciones del jaguar de la Sierra Madre Occidental, ya que esta área puede representar un eslabón entre Sahuaripa y la costa de Jalisco.

El conocimiento acerca de la ecología básica del jaguar es limitado para el estado de Sinaloa, ya que solo se ha publicado información anecdótica (Leopold 1959) y de distribución con base en registros de entrevistas (Navarro-Serment et al. 2005). Debido a la falta de información, nuestro objetivo fue estimar la abundancia y densidad de jaguar en el Área de Protección de Flora y Fauna Meseta de Cacaxtla (APFFM-Cacaxtla) ubicada en Sinaloa, México.

El APFFM-Cacaxtla (Fig. 1) se encuentra ubicada al sur del municipio San Ignacio y al norte del municipio de Mazatlán, tiene una extensión de $508.62 \mathrm{~km}^{2}$ y el rango de elevación va de los 0 a los $380 \mathrm{msnm}$ (DOF 2000). Los
Coronel-Arellano, H., Lara-Díaz, N. E., \& López-González, C. A. (2017) Jaguar (Panthera onca) abundance and density in the APFF Meseta de Cacaxtla, Sinaloa, Mexico. Acta Zoológica Mexicana (n.s.), 33(1), 116-119.

ABSTRACT. There is a lack of information about jaguar populations in Sinaloa, Mexico, because there are only anecdotic records and interviews about the presence of this felid. The aim of this study was to generate information about jaguar abundance and density in the Natural Protected Area Meseta de Cacaxtla. To detect jaguar, we used camera traps. The density obtained was 1.59 ind./100 $\mathrm{km}^{2}$ and 15 jaguars for the Natural Protected Area. We considered the Natural Protected Area as an important region for jaguar populations at the Sierra Madre Occidental, because this area may represent a linkage between Sahuaripa and Costa de Jalisco.

principales tipos de vegetación son selva baja caducifolia y selva mediana subcaducifolia; en menor cobertura se encuentran matorral xerófilo, esteros y lagunas (SEDUSU 2008). El clima es semicálido con lluvias en verano, con una temperatura media anual mayor a $\operatorname{los} 22^{\circ} \mathrm{C}$ y la precipitación anual promedio es de $700 \mathrm{~mm}$ (SEDESU 2008).

En el área de estudio llevamos a cabo tres prospecciones a campo: 1) del 12 de noviembre al 17 de diciembre de 2007, 2) del 18 de diciembre de 2007 al 20 de enero de 2008 y 3) del 24 de enero al 31 de marzo de 2008. Para la detección de la especie colocamos por prospección siete, diez y siete estaciones respectivamente, con una trampa- 


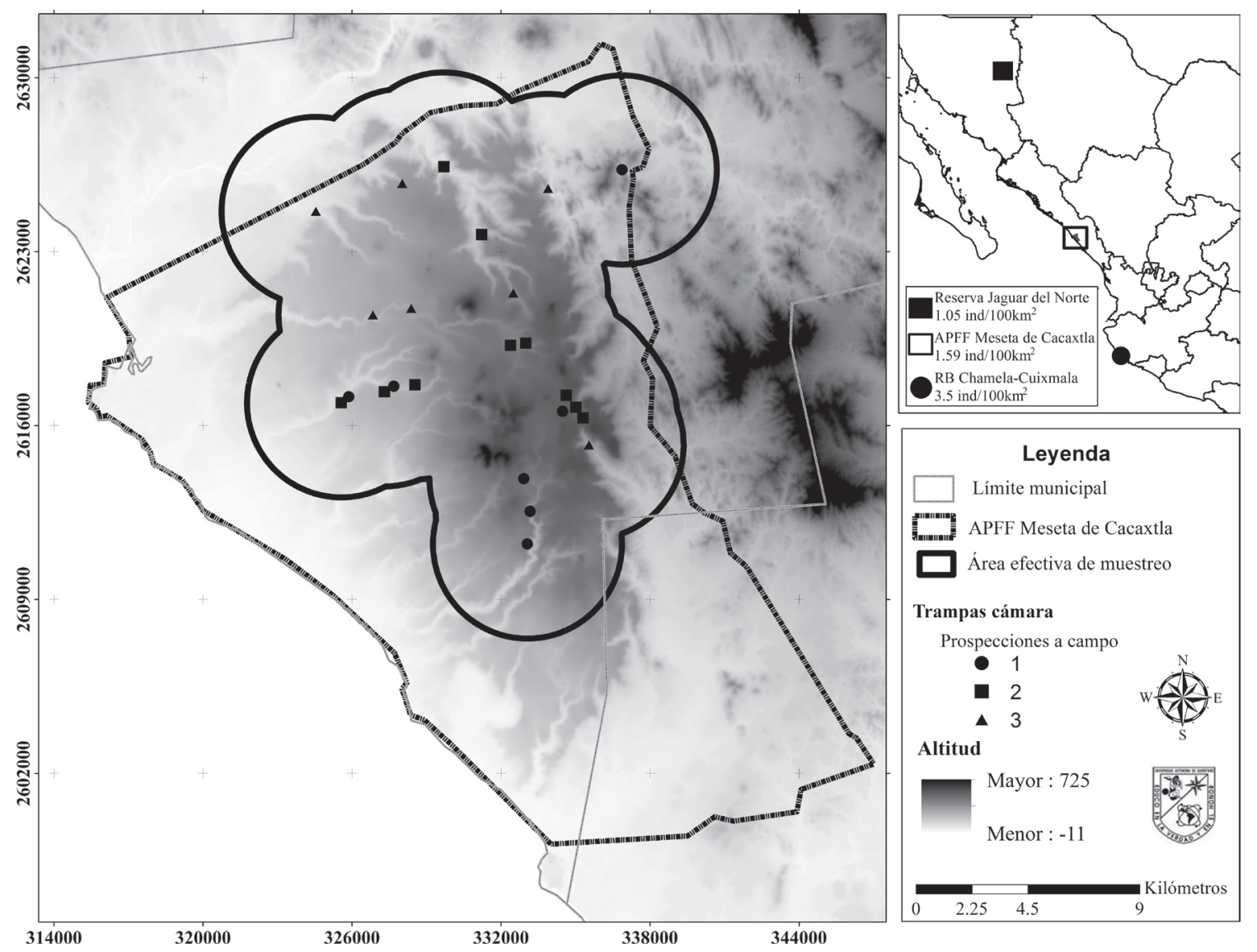

Figura 1. Localización geográfica del Área de Protección de Flora y Fauna Meseta de Cacaxtla, ubicación de trampas cámara y área efectiva de muestreo. Comparación de la densidad de jaguar en otras áreas protegidas cercanas.

cámara (Camtraker ${ }^{\circledR}$ ), en veredas identificadas como paso de fauna, con una orientación norte-sur para evitar que fueran activadas por la luz del sol. Las cámaras se fijaron a árboles a una altura entre 50 y $70 \mathrm{~cm}$. La distancia entre cámaras estuvo en el rango de uno a seis kilómetros.

Para estimar la abundancia construimos historias de captura utilizando las fotografías de jaguares obtenidas; los individuos fueron identificados con base en su patrón único de manchas (Gutiérrez-González et al. 2012). Se utilizó la extensión CAPTURE del programa MARK Versión 5.0 (White 2008) para obtener los valores de abundancia, para lo cual, cada prospección a campo fue considerada como una ocasión de muestreo. Para el cálculo de la abundancia se utilizaron tres modelos de población cerrada: Zippin como modelo de conducta $\left(\mathrm{M}_{\mathrm{b}}\right)$, y como modelos heterogéneos Jackknife $\left(\mathrm{M}_{\mathrm{h}}\right)$ y Chao $\left(\mathrm{M}_{\mathrm{h}}\right)$ (Lee \& Chao 1994). El área efectiva que utilizamos la obtuvimos generando un buffer circular alrededor de las trampas cámara, con un radio igual al promedio del ámbi- to hogareño $\left(45.5 \mathrm{~km}^{2}\right)$ de la especie reportado en Chamela, Jalisco (Núñez et al. 2002). La densidad fue estimada como ind $/ 100 \mathrm{~km}^{2}$ dividiendo la abundancia entre el área efectiva de muestreo y multiplicando el resultado por 100. La densidad fue extrapolada a la extensión de la APFFMeseta de Cacaxtla $\left(508.62 \mathrm{~km}^{2}\right)$, para tener una estimación de los individuos que podría tener esta reserva.

Con un esfuerzo de muestreo de 919 días-cámara, estimado como el producto del número de cámaras por los días que las cámaras permanecieron activas, obtuvimos cinco registros fotográficos de dos machos, dos hembras y un individuo sin sexo definido. Los registros corresponden a tres individuos de cuerpo completo y dos en los que solo se aprecian los cuartos traseros (Figura 2). Las historias de captura generadas fueron: jaguar macho $1-100$; jaguar hembra 2-100; jaguar sin sexo definido 3-010, jaguar macho 4-010 y jaguar hembra 5-001. Los tres modelos de abundancia estimaron cinco individuos (Zip$\operatorname{pin}=5 \pm 1.18, \mathrm{IC}=0$ a $10 ;$ Jackknife $=5 \pm 2.43, \mathrm{IC}=0$ 

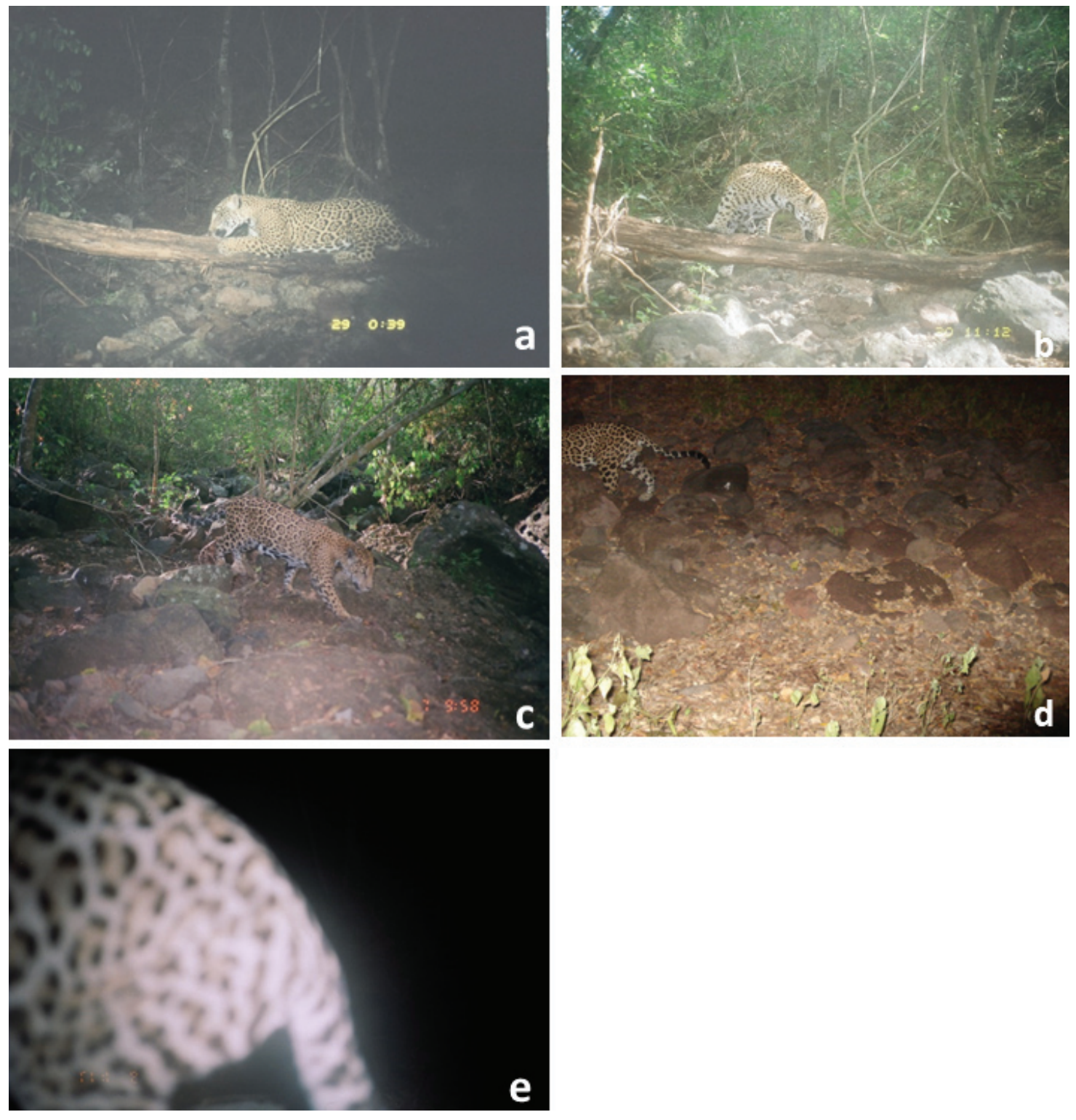

Figura 2. Registros fotográficos de jaguares obtenidos en tres prospecciones a campo en el Área de Protección de Flora y Fauna Meseta de Cacaxtla. Donde: a) = macho 1; b) hembra 1; c) = individuo sin sexo identificado; d) $=$ macho 2 ; e) $=$ hembra 2 .

a 15; Chao $=5 \pm 0, \mathrm{IC}=5$ a 5). Con un área efectiva de muestreo de $313.82 \mathrm{~km}^{2}$ obtuvimos una densidad de 1.59 ind $/ 100 \mathrm{~km}^{2}$ y ocho jaguares dentro del APFFM-Cacaxtla. La proporción de sexos identificados fue de 1:1.

El APFFM-Cacaxtla es reconocida como un área importante para la conservación de mamíferos de tamaño corporal grande (Morrison et al. 2007), lo cual es validado por nuestros resultados, ya que el área de estudio alberga una pequeña población reproductiva de jaguares (representada por las fotografías de hembras). Este es uno de los pocos estudios con jaguares donde la proporción de sexos es de uno a uno, en otros sitios tiende a presentarse una dominancia por machos (Foster et al. 2010, Palomares et al. 2012). Nosotros consideramos que el APFFMCacaxtla representa una unidad ecológica importante para las poblaciones de jaguar presentes en la Sierra Madre Occidental, ya que puede estar funcionando como un eslabón ("linkage" sensu Beier et al. 2006) en el intercambio de individuos que migren al norte en donde se encuentra la población reproductiva más norteña de este felino en 
Sahuaripa, Sonora (Gutiérrez-González et al. 2012) y al sur con las poblaciones de la Costa de Jalisco (Brown \& López-González 2001, Núñez et al. 2002, Sanderson et al. 2002; ver Fig. 1).

La densidad que obtuvimos en el APFFM-Cacaxtla (1.59 ind $/ 100 \mathrm{~km}^{2}$ ) es mayor a la reportada en la Reserva Jaguar del Norte, Sonora (1.05 ind $/ 100$ km²; Gutiérrez-González et al. 2012), y menor a la reportada en la Reserva de la Biosfera Chamela-Cuixmala, Jalisco (3.5 ind $/ 100 \mathrm{~km}^{2}$; Núñez et al. 2002). De acuerdo a la densidad de las tres reservas, la densidad de jaguares disminuye en relación al aumento de latitud. De este modo se podría generar un modelo lineal que pudiera ser usado para estimar una densidad de jaguares en ambientes con hábitats semejantes, como son las selvas caducifolias del Pacífico Mexicano. Finalmente, se ha determinado que Sinaloa forma parte de la distribución histórica y actual del jaguar, aún mantiene extensiones considerables de hábitat adecuado, e incluso se ha propuesto que puede mantener poblaciones con más de 100 individuos (Ceballos et al. 2006, Rabinowitz \& Zeller 2010, Rodríguez-Soto et al. 2011) por lo que es necesario llevar a cabo monitoreos a largo plazo para obtener estimaciones de abundancia y densidad más confiables, tanto en nuestra área de estudio como en otras regiones de la entidad.

AGRADECIMIENTOS. Al personal de la APFF Meseta de Cacaxtla y a los dueños de los predios en el Área de Protección por su valiosa ayuda e interés durante el trabajo de campo. Al proyecto Ecología del jaguar y su relación con la depredación de ganado en el Estado de Sonora y Querétaro (SEMARNAT-2002-C01-C388) por haber proporcionado el equipo de campo, así como a la Universidad Autónoma de Querétaro por el manejo administrativo de los recursos.

\section{LITERATURA CITADA}

Beier, P., Penrod, L. K., Luke, C., Spencer, D. W. \& Cabañero, C. 2006. South coast missing linkages: restoring connectivity to wildlands in the largest metropolitan area in the USA, Connectivity Conservation. Pp. 555-586. In: K. R. Crooks \& M. Sanjayan (Eds.). Connectivity Conservation. Cambridge University Press, New York, USA.

Brown, D. \& López González, C. 2001. Borderland Jaguars (Tigres de la Frontera). The University of Utah Press, Salt Lake City, USA, 170 pp.

Ceballos, G., Chávez, C., Blanco, S., Jiménez, R., López, M., Moctezuma, O., Támez, V.\& Valdez, M. 2006. Áreas prioritarias para la conservación. Pp. 13-19. In: C. Chávez \& G. Ceballos. (Eds.). Memorias del Primer Simposio. El Jaguar Mexicano en el Siglo XXI: Situación Actual y Manejo. CONABIO-Alianza WWF Telcel-Universidad Nacional Autónoma de México, México, D.F.
DOF 2000. Diario Oficial de la Federación Decreto por el que se declara área natural protegida, con el carácter de área de protección de flora y fauna, la región conocida como Meseta de Cacaxtla. Disponible en: http://dof.gob.mx/nota_detalle.php?codigo=69156 0\&fecha $=01 / 08 / 2003$ (accesado en septiembre 2009).

Foster, R. J., Harmsen, B. J. \& Doncaster, C. P. 2010. Habitat use by sympatric jaguars and pumas across a gradient of human disturbance in Belize. Biotropica, 42, 724-731.

Gutiérrez-González, C., Gómez-Ramírez, M. A. \& López-González, C. A. 2012. Estimation of the density of the Near Threatened jaguar Panthera onca in Sonora, Mexico, using camera trapping and an open population model. Oryx, 46, 431-437.

Lee, S. M. \& Chao, A. (1994. Estimating population size via simple coverage for closed capture-recapture models. Biometrics, 50, 88-97.

Leopold, A. 1959. Fauna silvestre de México. Instituto Mexicano de Recursos Naturales Renovables. Ciudad de México, México, 568 pp.

Morrison, C. J., Sechrest, W., Dinerstein, E., Wilcove, S. D. \& Lamoreux, J. 2007. Persistence of large mammal fauna as indicators of global human impacts. Journal of Mammalogy, 88, 13631380 .

Navarro-Serement, C.J., López-González, C. A. \& Gallo-Reynoso, J. P. (2005. Occurrence of jaguar (Panthera onca) in Sinaloa, México. The Southwestern Naturalist, 50, 102-106.

Núñez, R., Miller, B. \& Lindzey, F. 2002. Ecología del jaguar en la reserva de la biosfera Chamela-Cuixmala, Jalisco, México. Pp. 107-126. In: R. Medellín, C. Equihua, C. L. Chetkiewicz, P. G. Crawshaw, A. Rabinowitz, K. H. Redford, J. G. Robinson, W. Sanderson \& A. B. Taber (Eds.). El jaguar en el nuevo milenio. Fondo de Cultura Económica, Universidad Nacional Autónoma de México, Wildlife Conservation Society. México, D.F.

Palomares, F., Roques, S., Chávez, C., Silveira, L., Keller, C., Sollmann, R., do Prado, D. M., Torres, P.C., Adrados, B., Godoy, J. A. \& Jácomo, A. T. A. 2012. High proportion of male faeces in jaguar populations. PloS one, 7(12) e52923.

Rabinowitz, A. \& Zeller, K. A. 2010. A rage-wide model of landscape connectivity and conservation for the jaguar, Panthera onca. Biological Conservation, 143, 939-945.

Sanderson, W. E., Chetkiewicz, C. L. B., Medellín, A. R., Rabinowitz, A., Redford, H. K., Robinson, G. J. \& Taber, A. B. 2002. Prioridades geográficas para la conservación del jaguar. Pp. 601-627. In: R. Medellín, C. Equihua, C. L. Chetkiewicz, P. G. Crawshaw, A. Rabinowitz, K. H. Redford, J. G. Robinson, W. Sanderson \& A. B. Taber (Eds.). El jaguar en el nuevo milenio. Fondo de Cultura Económica, Universidad Nacional Autónoma de México, Wildlife Conservation Society. México, D.F.

SEDESU 2008. Secretaría de Desarrollo Social y Sustentable. Ordenamiento Ecológico Costero del Estado de Sinaloa. Disponible en: http://laip.sinaloa.gob.mx/LAIP/Secretaria/SPD/progserv/partciud/ORDENAMIENTO_ECOLOGICO_COSTERO_SIN.htm. (accesado en enero 2008).

White, C. G. 2008. Mark and Recapture Parameter Estimation. Version 5.0. Colorado State University. Disponible en: http://welcome.warnercnr.colostate.edu/ gwhite/mark/mark.htm (accesado en septiembre 2008). 